\title{
Combination of Episulfide Ring-Opening Polymerization With ATRP for the Preparation of Amphiphilic Block Copolymers
}

\author{
Cong-Duan Vo, Christopher J. Cadman, Roberto Donno, Jeroen A.C.M. Goos, \\ Nicola Tirelli*
}

We report for the first time the combination of ATRP and ring-opening episulfide polymerization as a means to synthesize polysulfide-based low-dispersity amphiphilic block copolymers. The most significant finding is the possibility to perform ATRP under mild conditions using poly(propylene sulfide) macroinitiators, apparently without any significant copper sequestration by the polysulfides. Using glycerol monomethacrylate (GMMA) as a hydrophilic monomer, the polymers self-assembled in colloidal structures with a morphology depending on the PS/GMMA ratio, but also probably on GMMA degree of polymerization. We here also present a new AFM-based method to calculate the average number of amphiphilic macromolecules per micelle.

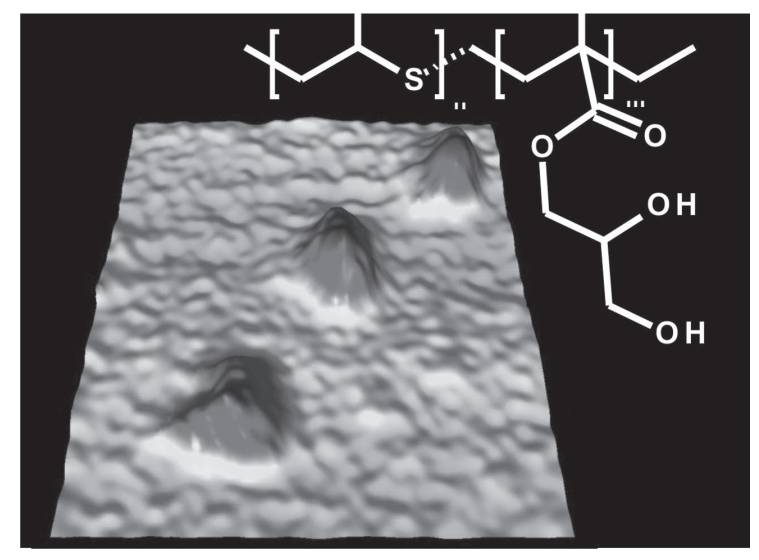

\section{Introduction}

Our group is heavily involved in the development of sulfur-based oxidation-responsive materials. In particular, we focus on poly(propylene sulfide) (PPS)-containing

C.-D. Vo, ${ }^{[+]}$N. Tirelli

School of Materials, University of Manchester, Manchester M13 9PT, UK

E-mail: Nicola.Tirelli@manchester.ac.uk

C. J. Cadman

School of Pharmacy and University of Manchester,

Manchester M13 9PT, UK

R. Donno, N. Tirelli

School of Medicine, University of Manchester, Manchester $M_{13}$ gPT, UK

J. A. C. M. Goos

Department of Pathology, VU University Medical Center, 1007 MB Amsterdam, the Netherlands

[+] Nanoco Technologies Ltd, 46 Grafton Street, Manchester M13 NT, UK. systems in the form of nanoparticles, ${ }^{[1,2]}$ vesicles, $^{[3]}$ or micelles, ${ }^{[4,5]}$ whose oxidative response is based on the conversion of hydrophobic thioethers to hydrophilic groups (depending on the oxidant, sulfoxides alone or in combination with sulfones ${ }^{[6]}$ ) and may lead to dramatic morphological changes. Most often, these colloidal structures are produced by amphiphilic block copolymers obtained through the conjugation of the hydrophobic PPS to hydrophilic poly(ethylene glycol)(PEG) derivatives, which also confer a biocompatible and "stealth" behavior. The weak points of this strategy are: (a) the possibility of varying the molecular weight of PEG: only a rather small number of PEG lengths are commercially available, while its ad hoc synthesis via anionic polymerization requires strictly anhydrous conditions and tolerates a limited number of functional groups, making the incorporation of biological species to polymers sometimes difficult; (b) PEG's inherently limited functionality, in comparison to, for example, polymers with functional side chains. The most logical alternative to the above is the preparation of hydrophilic blocks with functional side chains through a 
polymerization mechanism that offers good control over molecular weight, narrow molecular weight distribution, and tolerance to a variety of chemical groups. Atom transfer radical polymerization (ATRP) generally presents these features; in the perspective of an alternative to PEG glycerol monomethacrylate is polymerizable through ATRP ${ }^{[7]}$ and offers several interesting points: poly(glycerol monomethacrylate) (PGMMA) is very hydrophilic, it has been used as hydrophilic block in a variety of macromolecular amphiphilic structures, ${ }^{[8,9]}$ it shows minimal interactions with proteins ${ }^{[10]}$ or cell adhesion, ${ }^{[11]}$ and its alcohol groups can be readily functionalized (e.g., as anions, ${ }^{[12]}$ as hydrolytically cleavable moieties, ${ }^{[13]}$ as targeting groups). ${ }^{[14,15]}$

Although PPS-PGMMA block copolymers could be prepared by linking together two pre-formed blocks, the most convenient approach is a sequential polymerization of PS and GMMA. The question we have addressed in this study is whether a polysulfide chain may be used as an ATRP macroinitiator, since its rather high coordinating power may lead to significant alteration of the copper catalyst and to its possibly irreversible deactivation. This can happen due to irreversible complexation/ sequestration, as it has been invoked, for example, in the case of (meth)acrylamides, whose ATRP has always been marred by poor control ${ }^{[16-18]}$ with polydispersity values that only in rare cases have been below $1.5 ;{ }^{[19]}$ an indirect that these effects are caused by sequestration is that the polymerization of $\mathrm{N}$-isopropylacrylamide conducted under conditions of complete polymer solubility (low temperature) is well controlled. ${ }^{[20]}$ ATRP inhibition has also been recorded as an effect of $\mathrm{Cu}$ sequestration by carboxylic acids of monomers or of their polymerization products. ${ }^{[21]}$ However, the ATRP system may be affected by loss of deactivator due to reduced halidophilicity of the $\mathrm{Cu}$ (II) complexes; this is typically observed in a water environment and increases propagation rate and reduces the control over the polymerization process. ${ }^{[22]}$ DMSO shows a similar effect, which has been related to changes in the structure of ATRP catalysts by the coordination of DMSO to both $\mathrm{Cu}$ (II) and $\mathrm{Cu}$ (I) complexes, ${ }^{[23]}$ and later has been quantitatively explained on the basis of the effect of DMSO on halidophilicity of the oxidized copper complexes. ${ }^{[24]}$

In the present study, we have therefore tackled the synthesis of amphiphilic PPS-PGMMA diblock copolymers by using a bromoester-terminated PPS as a macroinitiator. Due to the hydrophobicity of PPS and its insolubility in most polar solvents, we have used GMMA in the form of its apolar acetonide derivative; the protected monomer, (2,2-dimethyl-1,3-dioxolan-4-yl)methyl methacrylate $(\mathrm{DMM})$, can be polymerized via anionic mechanism ${ }^{[25-27]}$ or ATRP ${ }^{[28,29]}$ and then hydrolyzed to restore the hydroxylated, hydrophilic GMMA block.

\section{Results and Discussion}

\subsection{Synthesis of Block Copolymers}

The description of all synthetic procedures is reported in the Supporting Information, Experimental Section.

\subsubsection{Monomer/End-Capper Synthesis}

DMM, the protected form of GMMA, was obtained via esterification of the alcohol group of 1,2-isopropylidene glycerol with methacryloyl chloride (Scheme 1; see also Experimental Section, and Figure S1, Supporting Information). PPS was converted into an ATRP macroinitiator by using an appropriate bifunctional reagent, $\mathbf{B i s B r}$, which is able to act both as a thiol-reactive end-capper for PPS and as an initiator for DMM ATRP.

The two bromoester groups with orthogonal reactivity were introduced functionalizing ethylene glycol first functionalized with a 2-bromoisobutyryl residue (ATRP-active, but un-reactive with thiols due to steric hindrance), and then introducing a thiol-reactive 2-bromoacetyl group (Scheme 1; see also Experimental Section and Figure S2, Supporting Information).

\subsubsection{Propylene Sulfide ROP}

Previous results have shown that the methanolysis of thioacetate precursors is a convenient method to initiate the episulfide ring opening polymerization (ROP): in combination with the use of an in situ reducing agent (tributyl phosphine) this method allows to minimize the presence of disulfides, ${ }^{[30]}$ which act as chain transfer agent in this polymerization. ${ }^{[31]}$ However, in this case, we have noticed that excess methanol used in the deprotection procedure affected the yield of PPS end-capping $(<70-80 \%)$ by reacting with the 2-bromoacetyl group in BisBr. We have therefore employed the initiator in the form of free thiol; despite of the presence of the reducing agent, this caused an increase in polydispersity (1.22 vs 1.15 for degree of polymerization $=30$ ), but allowed for an almost quantitative end-capping (95-98\% mol), due to the absence of nucleophilic species competing with thiolates. The presence of non end-capped, disulfide-containing polysulfide homopolymers, (Figure 1A) is estimated at about $5 \mathrm{wt} \%$; due to the absence of terminal bromoester groups, these macromolecules are supposedly left untouched during ATRP.

\subsubsection{ATRP and Deprotection}

DMM ATRP has been conducted in aromatic/high-boiling solvents, typically providing rather high polydispersity values: $\bar{M}_{\mathrm{w}} / \bar{M}_{\mathrm{n}}>1.4$ for 1,2 dichlorobenzene at $85^{\circ} \mathrm{C}$ (solution, 2-bromopropionitrile as initiator and $\mathrm{CuBr}_{2} / \mathrm{HMTETA}^{\circ}$

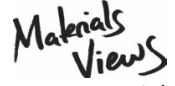

www.MaterialsViews.com 
<smiles>CCCCC(C)(C)C(=O)OCCOC(=O)CBr</smiles>

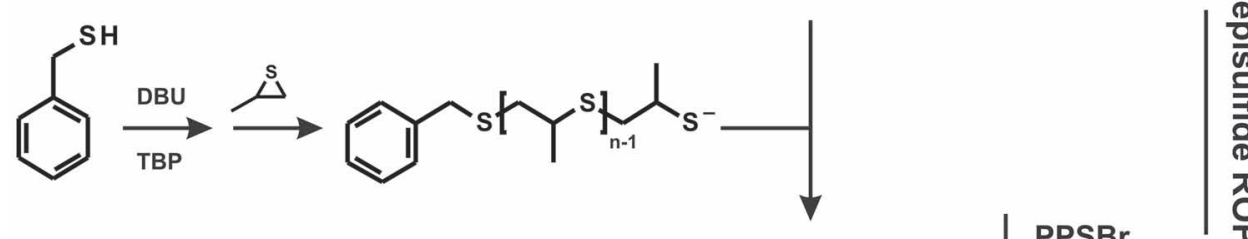<smiles>C=C(C)C(=O)OCC1COC(C)(C)O1</smiles>

Scheme 1. Benzyl mercaptan was deprotonated by DBU and then used to initiate the ring-opening polymerization of propylene sulfide, using tributyl phosphine (TBP) as an in situ reducing agent; the thiolate-terminated polysulfide chains ( $n=30$ or 39 in this study) were end-capped with the double functional reagent $\mathrm{Bis} B \mathrm{~B}$, which was previously prepared from ethylene glycol through successive esterification reactions with two different 2-bromoacetyl bromides. The resulting end-capped polymers bore a terminal ATRP initiator; they were then employed to polymerize an ad hoc prepared monomer (DMM), which was obtained through the methacrylation of 1,2-isopropylidene glycerol. The corresponding block copolymers were synthesized with $\mathrm{m} / \mathrm{n}$ ratios in the range $0.45-2.6$ (see Table S1, Supporting Information). Finally, the hydrophobic diblock copolymers were treated with formic acid to uncover diol groups and convert them into amphiphilic structures.

as catalyst), ${ }^{[28]} \bar{M}_{\mathrm{w}} / \bar{M}_{\mathrm{n}}=1.3-1.4$ for isobutyl acetate at $40{ }^{\circ} \mathrm{C}$ (solution, $\mathrm{Br}-\mathrm{PPO}_{34}-\mathrm{Br}$ as macroinitiator and $\mathrm{CuCl} /$ $\mathrm{CuCl}_{2} /$ bpy as catalyst).[29] Similar conditions were also applied to DMM surface-initiated ATRP: anisole at $60{ }^{\circ} \mathrm{C}$ (CuBr/spartein as catalyst). ${ }^{[32]}$ In DMM homopolymerization (initiator: ethyl 2-bromoisobutyrate (EBIB)), we have obtained excellent results in THF, with first-order kinetics both at 25 or $60{ }^{\circ} \mathrm{C}$ (Figure 1B); the molecular weight increased with monomer conversion while maintaining a polydispersity index always $<1.2$ (Figure $1 \mathrm{C}$; see also Figure S4; Supporting Information).
We have applied the same conditions to PPS macroinitiators. Very slow DMM conversion ( $\leq 20 \%$ after $24 \mathrm{~h}$ ) and bimodal GPC curves (not shown) were recorded at $25^{\circ} \mathrm{C}$; these effects can be due to catalyst deactivation due to halidophilicity of the polysulfide chain, although we cannot exclude poor solubility of the macroinitiators to play a role. On the other hand, these effects were not recorded at $60^{\circ} \mathrm{C}$ : monomer conversion and polydispersity followed virtually the same kinetics as in the homopolymerization experiments, which may suggest that a significant temperature dependency of the copper/polysulfide interactions. 

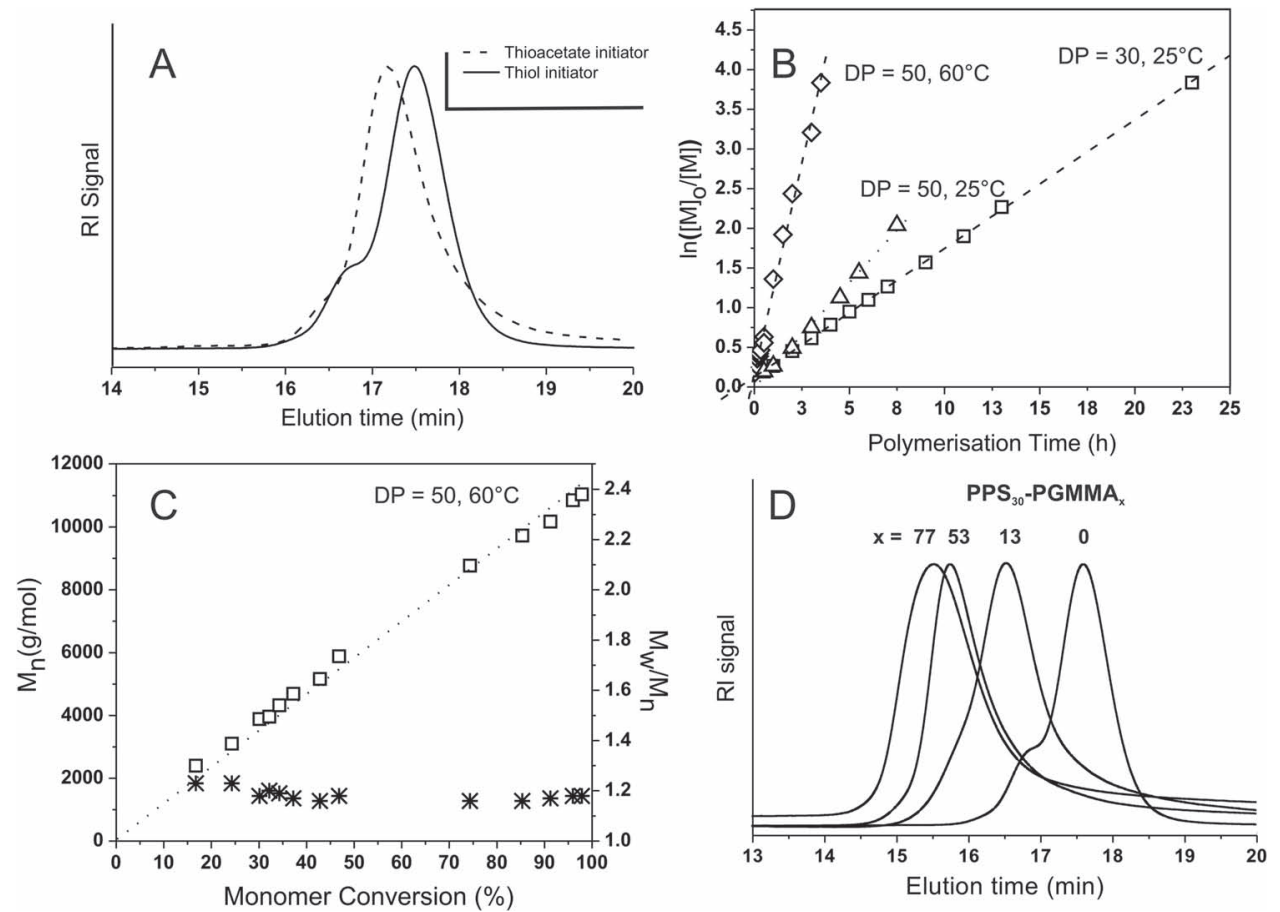

Figure 1. A: GPC traces of $\mathrm{PPS}_{30}$ produced using benzyl thioacetate (see Figure $3 \mathrm{SI}$; Supporting Information, SI, left) or benzyl mercaptan as initiators [THF, ref. index detector, polystyrene standards]. Due to the presence of disulfide impurities, in the second case, the distribution is slightly shifted to lower molecular weight values, while a shoulder appears on its left (dimer shoulder, which correspond to polysulfide chains with internal disulfide bond). See also Supporting Information, Figure S3. B: DMM conversion (from ${ }^{1} \mathrm{H} N M R$ ) in three homopolymerization experiments performed using different DMM:EBIB ratios (theoretical DP) and different temperatures; catalyst: $\mathrm{CUCl} / \mathrm{HMTETA}$. C: Number-average molecular weight and polydispersity index (from GPC in THF) versus monomer conversion (from ${ }^{1} \mathrm{H}$ NMR) during ATRP at $60{ }^{\circ} \mathrm{C}$ (theoretical DP $=50$ ). $D$ : from right to left, GPC (THF) traces of $\mathrm{PPS}_{30}$, and of acetylated $\mathrm{PPS}_{30}-\mathrm{PGMMA}_{13}, \mathrm{PPS}_{30}-\mathrm{PGMMA}_{53}$ and PPS $30^{-}$ $\mathrm{PGMMA}_{77}$. The latter curves are almost perfectly superimposable to those of the corresponding PPS/PDMM copolymers before deprotection, but lack the peaks associated to polysulfide dimers; the latter were removed during the purification after GMMA deprotection (see Figure S4, Supporting Information).

The PPS-PDMM block copolymers were deprotected with $80 \%$ formic acid in water at $20{ }^{\circ} \mathrm{C}$ for 2 days: ${ }^{1} \mathrm{H}$ NMR (Figure 2A), ${ }^{13} \mathrm{C}$ NMR (Figure 2B), and IR (Figure 2C) showed the complete disappearance of the signals of the acetonide ring and the appearance of alcohols. The resulting PPSPGMMA block copolymers showed unimodal molecular weight distribution, reasonably narrow polydispersity $(<1.25)$ and a linear relation between molecular weight and the DMM/initiator ratio (Figure 1D).

It is noteworthy that the polydispersity of the protected block copolymers was considerably higher than those of the parent PPS, of PDMM controls and also of the deprotected polymers: $\bar{M}_{\mathrm{w}} / \bar{M}_{\mathrm{n}}=1.28-1.39$ for PPS-PDMM, versus 1.22 for PPS, 1.12-1.25 for PDMM homopolymers and 1.18-1.26 for PPS-PGMMA (see Table S1, Supporting Information). This was due to the presence of non-functional PPS; these homopolymers did not participate to the ATRP and could be eventually removed from the amphiphilic materials via extraction with hexane (see Figure S5, Supporting Information).

\subsection{Self-Assembly in Water}

All PPS-PGMMA block copolymers, and above all those with low GMMA content (e.g., $\mathrm{PPS}_{30}$ PGMMA $_{13}$ and $\mathrm{PPS}_{39}$-PGMMA $\mathrm{P}_{23}$ ), provided large aggregates with broad size distributions (300 $\mathrm{nm}$ to micron range) when dispersed in deionized water. It is reasonable to assume this behavior to be due to hydrogen bonding between OH-rich blocks; as in the case of poly(2-hydroxypropyl methacrylate) copolymers, ${ }^{[33]}$ the use of sodium nitrate as an hydrogen bonding-disrupting agent allowed to obtain smaller aggregates, whose size was unchanged by either increasing nitrate concentration (5 to $10 \times 10^{-3} \mathrm{M}$ ) or reverting to deionized water after dialysis. The hydrodynamic radius of the resulting colloids depended quite sharply on the hydrophobic/hydrophilic balance (expressed as PS/GMMA molar ratio in Figure 3A) of the polymers; larger aggregates were recorded for the more hydrophobic polymers, which is not surprising since bulkier hydrophobes typically drive the assembly toward

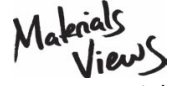

www.MaterialsViews.com 

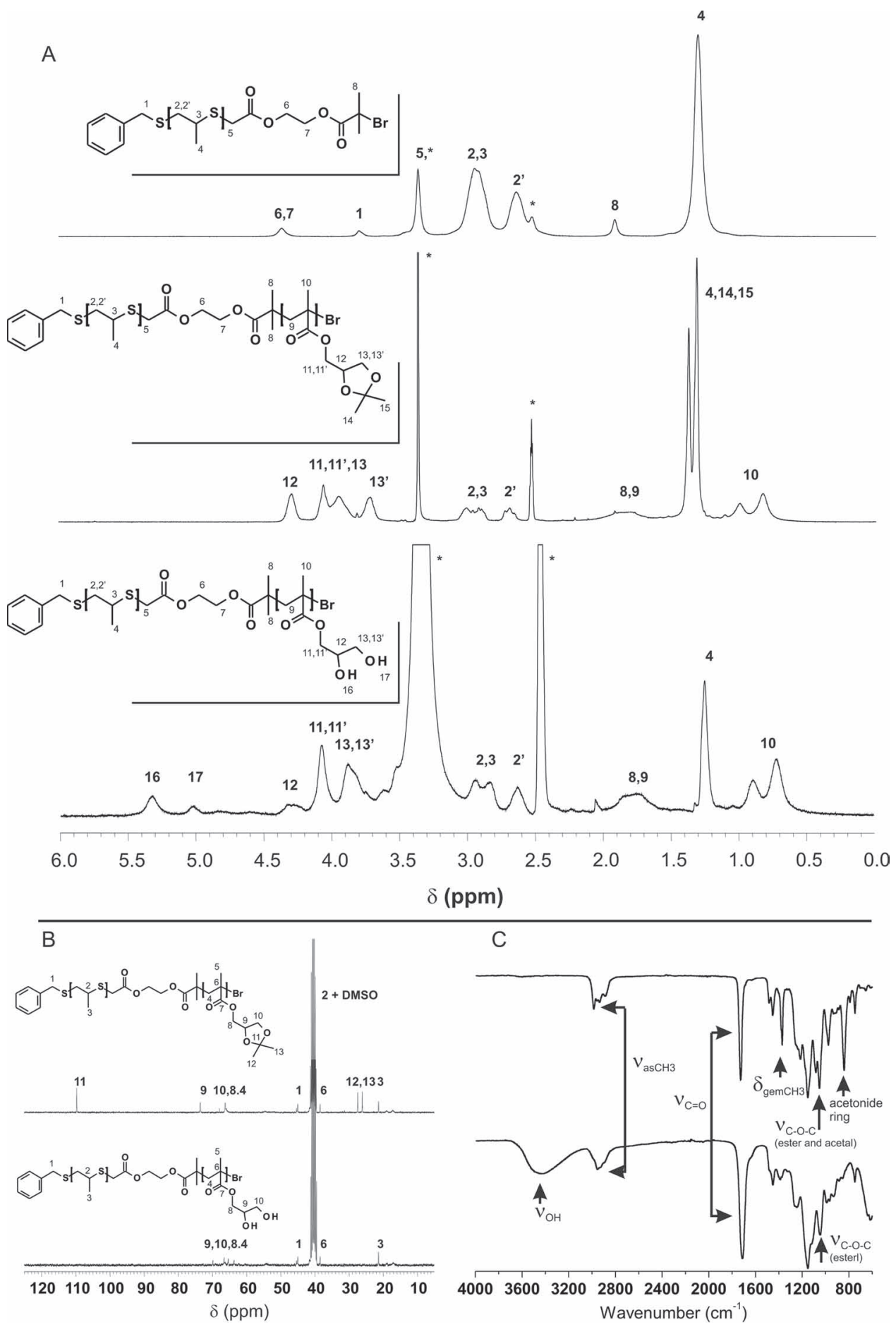

Figure 2. $A:{ }^{1} \mathrm{H}$ NMR spectra of $\mathrm{PPS}_{30}$ (top) $\mathrm{PPS}_{30}-\mathrm{PGMMA}_{53}$ (middle) and $\mathrm{PPS}_{30}-\mathrm{PGMMA}_{53}$ (bottom). Please note that in the latter case the large water peak was due to the hydrophilicity of GMMA, which could not be effectively dried even via toluene azeotropic distillation; in all the spectra, the peaks of water and DMSO are highlighted with stars; the degrees of polymerization are omitted in the formulae to avoid confusion with $\mathrm{H}$ atom numbering. The polymerization of DMM added signals typical of methacrylic chains (methyl group 10), of glycerol (protons 11 to 13) and of the acetonide ring (methyl groups 14 ad 15). The latter were no longer present after hydrolysis. B: ${ }^{13} \mathrm{C}$ NMR spectra of $\mathrm{PPS}_{30}-\mathrm{PGMMA}_{53}$ (top) and $\mathrm{PPS}_{30}-\mathrm{PGMMA}_{53}$ (bottom); formic acid caused the disappearance of all signals associated to the isopropylidene group (11,12 and 13). C: the diagnostic bands of the acetonide ring highlighted in the IR spectrum of $\mathrm{PPS}_{30}-\mathrm{PGMMA}_{53}$ (top) disappeared in that of $\mathrm{PPS}_{30}-\mathrm{PGMMA}_{53}$ (bottom), while a broad $\mathrm{vOH}$ band appeared above $3000 \mathrm{~cm}^{-1}$. The $1082 \mathrm{~cm}^{-1}$ component of the $\mathrm{VC}-\mathrm{O}-\mathrm{C}$ band (acetal) disappeared, while that at $1058 \mathrm{~cm}^{-1}$ (ester) was still present after deprotection. ${ }^{[29]}$ 


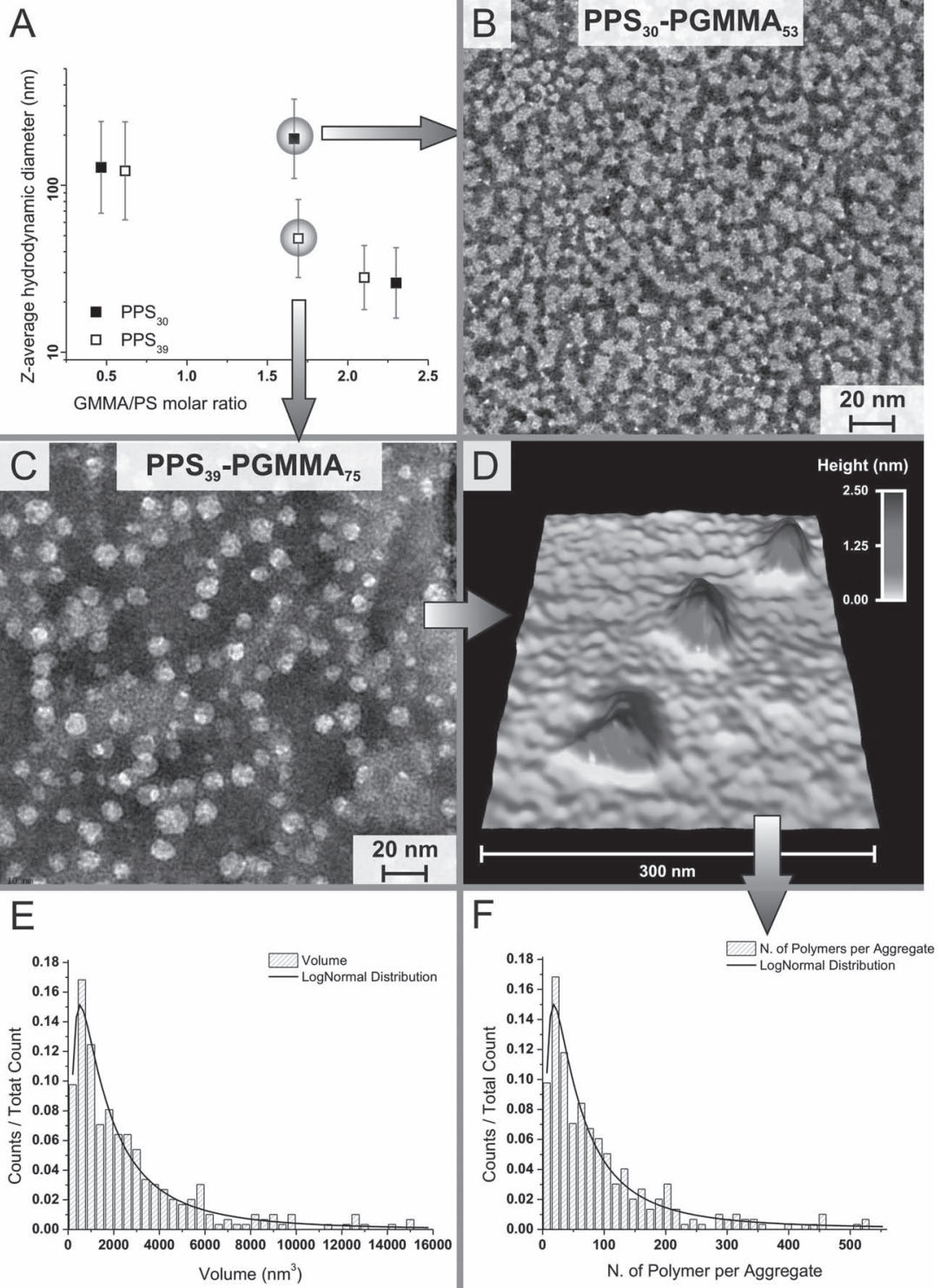

Figure 3. A: Z-average size (from DLS measurements) for different PPS-PGMMA copolymers with different PS/GMMA ratios and two different PS degrees of polymerization. The bars represent the width at half height of the peaks. $B$ : negative staining TEM picture for $\mathrm{PPS}_{30}-\mathrm{PGMMA}{ }_{53}$, showing the presence of irregular elongated structures (probably worm-like micelles). $C$ : negative staining TEM picture for $\mathrm{PPS}_{39}-\mathrm{PGMMA}_{75}$, showing the presence of spherical structures (spherical micelles). D: AFM image of $\mathrm{PPS}_{39}-\mathrm{PGMMA}_{75}$ aggregates deposited on mica. See also Figures $\mathrm{S} 6$ and $\mathrm{S} 7$, Supporting Information. $E$ : Number distribution of the volumes of $\mathrm{PPS}_{39}-\mathrm{PGMMA}_{75}$ aggregates, obtained from thresholding and segmenting the AFM images; the solid line is the result of a lognormal fitting. F: Number distribution of the number of polymer chains per aggregate, obtained by converting the volumes of the distribution in panel $\mathrm{E}$ first into masses and then into a moles of block copolymers; the solid line is the result of a lognormal fitting. different in size (see circled symbols in Figure $3 \mathrm{~A}$ ) that were also different in morphology: $\mathrm{PPS}_{30} \mathrm{PGMMA}_{53}$ gave rise to larger and predominantly elongated structures (Figure 3B), which could be interpreted as transitional/ aggregated states possibly preluding to worm-like micelles. On the contrary, $\mathrm{PPS}_{39} \mathrm{PGMMA}_{75}$ produced welldefined spherical micelles (Figure $3 \mathrm{C}$ ). We hypothesize this to stem from increasing hydration of GMMA units with increasing PGMMA size, as it happens for PEG. PPS ${ }_{30} \mathrm{PGMMA}_{75}$ in water presented a hydrodynamic diameter of about 30-60 nm (DLS).

Under vacuum, this was reduced to 10-15 nm (TEM), probably due to the loss of PGMMA hydration.

Under air and on a mica surface, these aggregates adopted a flattened morphology, due to the low $T_{\mathrm{g}}$ of the hydrophobic core (Figure 3D, see also Figures S6 and S7; Supporting Information). As described in Supporting Information, AFM analysis, we have then used a segmentation algorithm for the automatic recognition of the particles, employing the root mean square roughness of the substrate as a threshold. This allowed to calculate the volume of each individual aggregate and to produce an overall number distribution (Figure 3E), which was then analyzed with several statistical functions; the Akaike Information Criterion showed that best fits were obtained with a lognormal distribution, which provided a micellar number average volume of $2513 \mathrm{~nm}^{3}$; in a spherical morphology, this would correspond to a number average diameter of $16.8 \mathrm{~nm}$, whose similarity to TEM results is probably due again to the limited hydration of the PGMMA blocks in air. Neglecting therefore the presence of water and using an estimated value for the volume of $\mathrm{PPS}_{30} \mathrm{PGMMA}_{75}\left(28.2 \mathrm{~nm}^{3}\right.$, structures that are larger due to their lower surface/ volume ratio, such as lamellae or worm-like micelles. However, it is noteworthy that two polymers with very similar PS/GMMA ratio but different PS degree of polymerization (30 vs 39 ) produced colloids considerably see Supporting Information, AFM analysis) it was finally possible to convert the volume distribution in that of number of chains (Figure 3F), allowing therefore to estimate the most probable number of macromolecules per aggregate as $N_{\text {mac }}=99$.

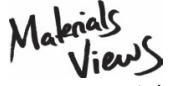

www.MaterialsViews.com 


\section{Conclusions}

Polysulfides can be integrated in ATRP processes, without significant interference in the polymerization and producing block copolymers with low polydispersity. Further, using the facile protection/deprotection of GMMA, it was possible to prepare amphiphilic copolymers, whose self-assembly in water produced morphologies controlled by both the PS/ GMMA ratio and the overall length of the polymer. Finally, we have demonstrated that AFM allows an assessment of the number of amphiphilic components per aggregate.

\section{Supporting Information}

Supporting Information is available from the Wiley Online Library or from the author.

Acknowledgements: The authors gratefully acknolwedge the Knoledge Centre of Materials Chemistry (KCMC) of the University of Manchester for financial contributions and want to thank Dr. Piero de Leonardis for acquiring some of the original AFM pictures and Dr. Ghislaine Robert-Nicoud for useful discussions.

Received:September 21, 2012; Revised: October 10, 2012; Published online: November 9, 2012; DOI: 10.1002/marc.201200636

Keywords: atomic force microscopy; block copolymers; micelles; polysulfides

[1] V. V. Khutoryanskiy, N. Tirelli, Pure Appl. Chem. 2008, 80, 1703.

[2] A. Rehor, H. Schmoekel, N. Tirelli, J. A. Hubbell, Biomaterials 2008, 29, 1958.

[3] A. Napoli, M. Valentini, N. Tirelli, M. Muller, J. A. Hubbell, Nat. Mater. 2004, 3, 183.

[4] P. Hu, N. Tirelli, React. Funct. Polym. 2011, 71, 303.

[5] P. Hu, N. Tirelli, Bioconjugate Chem. 2012, 23, 438.

[6] P. Carampin, E. Lallana, J. Laliturai, S. C. Carroccio, C. Puglisi, N. Tirelli, Macromol. Chem. Phys. 2012, 213, 2052.

[7] M. Save, J. V. M. Weaver, S. P. Armes, P. McKenna, Macromolecules 2002, 35, 1152 .

[8] A. Blanazs, R. Verber, O. O. Mykhaylyk, A. J. Ryan, J. Z. Heath, C. W. I. Douglas, S. P. Armes, J. Am. Chem. Soc. 2012, 134, 9741.
[9] K. L. Thompson, S. P. Armes, D. W. York, J. A. Burdis, Macromolecules 2010, 43, 2169.

[10] F. C. Giacomelli, P. Stepanek, V. Schmidt, E. Jaeger, A. Jaeger, C. Giacomelli, Nanoscale 2012, 4, 4504.

[11] E. Patrucco, S. Ouasti, C. D. Vo, P. De Leonardis, A. Pollicino, S. P. Armes, M. Scandola, N. Tirelli, Biomacromolecules 2009, 10, 3130.

[12] C. D. Vo, P. D. Iddon, S. P. Armes, Polymer 2007, 48, 1193.

[13] D. E. Discher, A. Eisenberg, Science 2002, 297, 967.

[14] D. E. Discher, V. Ortiz, G. Srinivas, M. L. Klein, Y. Kim, C. A. David, S. S. Cai, P. Photos, F. Ahmed, Progr. Polym. Sci. 2007, 32, 838.

[15] V. P. Torchilin, R. Rammohan, V. Weissig, T. S. Levchenko, Proc. Natl. Acad. Sci. USA 2001, 98, 8786.

[16] J. T. Rademacher, R. Baum, M. E. Pallack, W. J. Brittain, W. J. Simonsick, Macromolecules 2000, 33, 284.

[17] M. Teodorescu, K. Matyjaszewski, Macromol. Rapid Commun. 2000, 21, 190.

[18] S. K. Jewrajka, B. M. Mandal, Macromolecules 2003, 36, 311.

[19] D. A. Z. Wever, P. Raffa, F. Picchioni, A. A. Broekhuis, Macromolecules 2012, 45, 4040.

[20] P. E. Millard, N. C. Mougin, A. Boker, A. H. E. Muller, in Controlled/Living Radical Polymerization: Progress in ATRP, (Ed: K. Matyjasewski), American Chemical Society, Washington 2009, p. 127.

[21] A. Y. Sankhe, S. M. Husson, S. M. Kilbey, Macromolecules 2006, 39, 1376.

[22] N. V. Tsarevsky, W. A. Braunecker, A. Vacca, P. Gans, K. Matyjaszewski, Macromol. Symp. 2007, 248, 60.

[23] S. Monge, V. Darcos, D. M. Haddleton, J. Polym. Sci., Polym. Chem. 2004, 42, 6299.

[24] W. A. Braunecker, N. V. Tsarevsky, A. Gennaro, K. Matyjaszewski, Macromolecules 2009, 42, 6348.

[25] F. T. Liu, G. J. Liu, Macromolecules 2001, 34, 1302.

[26] H. Mori, A. Hirao, S. Nakahama, Macromolecules 1994, 27, 35.

[27] H. Mori, A. Hirao, S. Nakahama, K. Senshu, Macromolecules 1994, 27, 4093.

[28] Z. G. Zhang, G. J. Lin, S. Bell, Macromolecules 2000, 33, 7877.

[29] E. Amado, C. Augsten, K. Mader, A. Blume, J. Kressler, Macromolecules 2006, 39, 9486.

[30] G. Kilcher, L. Wang, N. Tirelli, J. Polym. Sci., Polym. Chem. 2008, 46, 2233.

[31] L. Wang, G. Kilcher, N. Tirelli, Macromol. Chem. Phys. 2009, $210,447$.

[32] M. Kobayashi, A. Takahara, Chem. Lett. 2005, 34, 1582.

[33] C. D. Vo, J. Rosselgong, S. P. Armes, N. Tirelli, J. Polym. Sci. Polym. Chem. 2010, 48, 2032. 